\title{
Neural Correlates of Cat Odor-Induced Anxiety in Rats: Region-Specific Effects of the Benzodiazepine Midazolam
}

\author{
Iain S. McGregor, ${ }^{1}$ Garth A. Hargreaves, ${ }^{1}$ Raimund Apfelbach, ${ }^{2}$ and Glenn E. Hunt ${ }^{3}$ \\ ${ }^{1}$ School of Psychology, University of Sydney, Sydney, New South Wales 2006, Australia, ${ }^{2}$ Tierphysiologie, Universitat Tubingen, D-72076 Tubingen, \\ Germany, and ${ }^{3}$ Department of Psychological Medicine, University of Sydney, Concord Hospital, Sydney, New South Wales 2139, Australia
}

Cat odor elicits a profound defensive reaction in rats that is reduced by benzodiazepine drugs. The neural correlates of this phenomenon were investigated here using Fos immunohistochemistry. Rats received either midazolam $(0.75 \mathrm{mg} / \mathrm{kg}$, s.c.) or vehicle and were exposed to pieces of a collar that had been worn by a domestic cat or an unworn (dummy) collar. Cat odor caused midazolam-sensitive defensive behavioral responses, including avoidance of collar contact, inhibition of grooming, and prolonged rearing. Cat odor exposure induced Fos expression in the posterior accessory olfactory bulb (glomerular, mitral, and granule cell layers), with granule cell layer activation attenuated by midazolam. High basal Fos expression, and some cat odor-associated Fos expression, was evident in the main olfactory bulb (glomerular cell layer), and midazolam exerted a strong inhibitory effect in this region. Midazolam inhibited Fos expression in key limbic regions involved in pheromone transduction (medial amygdala and bed nucleus of the stria terminalis) and defensive behavior (prelimbic cortex, lateral septum, lateral and medial preoptic areas, and dorsal premammillary nucleus). However, midazolam failed to affect cat odor-related Fos expression in a range of key defense-related sites, including the ventromedial hypothalamic nucleus, paraventricular nucleus of the hypothalamus, periaqueductal gray, and cuneiform nucleus. These results indicate that midazolam exerts a region-specific effect on the neural substrates activated by predator odor, with effects in the lateral septum and dorsal premammillary nucleus likely to be of major importance. These findings also suggest the intriguing hypothesis that cat odor is processed by rats as a "pheromone-like" stimulus.

Key words: odor; midazolam; anxiety; defense; predator; pheromone

\section{Introduction}

Several research groups have reported the fascinating phenomenon whereby rats show an innate defensive response to the odor of predators, particularly cats (Blanchard et al., 1990; Zangrossi and File, 1992; Dielenberg et al., 1999; Li et al., 2004). On encountering cat odor, rats show retreat, hiding, and a variety of risk assessment behaviors directed toward the monitoring of the predatory stimulus (Blanchard et al., 1990; Zangrossi and File, 1992; Dielenberg et al., 1999). This defensive response to cat odor is modulated by a number of different drugs, with a dramatic effect evident with benzodiazepines (Dielenberg et al., 1999; McGregor and Dielenberg, 1999; McGregor et al., 2002). Rats encountering cat odor for the first time while under the influence of midazolam show an absence of retreat, a lack of hiding, and altered risk assessment (Dielenberg et al., 1999; McGregor and Dielenberg, 1999; McGregor et al., 2002). This profound modulatory effect on defensive behavior in rodents may be relevant to

Received Jan. 17, 2004; revised Feb. 27, 2004; accepted March 4, 2004.

This work was supported by an Australian Research Council grant to I.S.M. We gratefully acknowledge fruitful discussions with Robert Dielenberg and the technical assistance of Murray Thompson and Ljiljana Sokolic.

Correspondence should be addressed to lain S. McGregor, School of Psychology, University of Sydney, A19, Sydney, New South Wales 2006, Australia. E-mail: iain@psych.usyd.edu.au.

DOI:10.1523/JNEUROSCI.0187-04.2004

Copyright $\odot 2004$ Society for Neuroscience $\quad$ 0270-6474/04/244134-11\$15.00/0 our understanding of the anti-anxiety actions of benzodiazepine drugs in humans (Gray and McNaughton, 2000; Blanchard et al., 2001).

In the present study, we determined Fos expression in rats exposed to cat odor with or without pretreatment with the benzodiazepine midazolam. Previously, we described a network of brain regions that is activated in rats encountering cat odor, which includes the medial amygdala, the bed nucleus of the stria terminalis (BNST), various medial hypothalamic structures, and the periaqueductal gray (PAG) (Dielenberg et al., 2001b). The regions are remarkably similar to those activated during encounters with a live cat (Canteras et al., 1997; Canteras and Goto, 1999). Complementary lesion studies have verified a key role of some of these regions in encounters with predators and predatorrelated stimuli (Canteras et al., 1997; Blanchard et al., 2003; Li et al., 2004).

One unresolved issue concerns the olfactory regions activated by cat odor in rats. Some have suggested that cat odor may engage the accessory olfactory (pheromone) system rather than the main olfactory system (Panksepp, 1998; Blanchard and Blanchard, 1999; Dielenberg et al., 2001b). Activation in olfactory structures could not be readily discerned in our previous study because of the use of a behavioral paradigm in which rats hid for long periods at some distance from the cat odor stimulus, thus minimizing olfactory stimulation (Dielenberg et al., 2001b). Accordingly, in the current experiment, we presented the cat odor stimulus in a 
confined environment in which escape from the stimulus was not readily achieved.

The anxiolytic action of midazolam in rats exposed to cat odor most likely involves an action on key limbic and brainstem regions mediating fear, anxiety, and defensive behavior. This hypothesis is supported by observations that injection of benzodiazepines into septal, amygdala, hypothalamic, and brainstem sites have pronounced effects in animal models of anxiety (for review, see Menard and Treit, 1999). However, midazolam could also conceivably affect sensory mechanisms involved in the transduction of the cat odor stimulus. If this were the case, then a modification of olfactory Fos expression to cat odor might be expected.

\section{Materials and Methods \\ Subjects}

The subjects $(n=23)$ were male Wistar rats, bred in our colony, and weighing $321 \pm 7 \mathrm{gm}$ at the time of testing. They were housed in groups of five or six with access to food and water ad libitum in a temperaturecontrolled colony room $\left(21 \pm 2^{\circ} \mathrm{C}\right)$ on a reverse light/dark cycle (lights on from 8:00 A.M. to 8:00 P.M.). All behavioral testing occurred during the dark cycle. All efforts were made to minimize both the suffering and the number of rats used. All experimentation was approved by the University of Sydney Animal Ethics Committee in accordance with the Australian Code of Practice for the Care and Use of Animals for Scientific Purposes.

\section{Apparatus}

The test apparatus comprised eight small enclosed rectangular chambers $(25 \times 13 \times 32 \mathrm{~cm})$ with three Perspex walls, one wooden wall, and a metal grid floor. The ceiling consisted of wire mesh. In the center of three of the walls, there was a metallic alligator clip located $4 \mathrm{~cm}$ above the metal grid floor. The clip was used to secure pieces of fabric collar to the side of the chamber. The test chamber was raised on legs over a removable drop tray that was cleaned thoroughly between test sessions. This apparatus differs from the much larger arena used in our previous studies in which rats had the opportunity to hide inside a small wooden hide box within a large test chamber (Dielenberg et al., 1999, 2001a,b; McGregor et al., 2002).

A miniature infrared-sensitive video camera was mounted on the wire mesh ceiling of the chamber to allow monitoring of behavior. The signal from the camera was recorded to tape for subsequent scoring.

The cat collars (Kra-mar stretch collar, model $08010 ; 13 \mathrm{~mm}$ width, 5 $\mathrm{mm}$ thickness, $200 \mathrm{~mm}$ length) had been worn by a domestic cat for a period of at least 2 weeks. The collar was cut into $25 \mathrm{~mm}$ pieces, placed in an air-tight plastic container, and stored at $-10^{\circ} \mathrm{C}$. The collar pieces were handled with latex disposable gloves and warmed in a sealed glass jar placed in a sink of hot water before being attached to the alligator clips.

\section{Procedure}

Rats were randomly assigned to one of four groups: CAT-SAL $(n=7)$, CAT-MDZ $(n=6)$, CONTROL-SAL $(n=6)$, and CONTROL-MDZ $(n=4)$. Rats in the two CAT groups were exposed to a worn cat collar on test day, whereas rats in the two CONTROL groups were exposed to an equivalent unworn collar. Rats in the two MDZ groups were injected subcutaneously with midazolam $(0.75 \mathrm{mg} / \mathrm{kg}$ in a volume of $1 \mathrm{ml} / \mathrm{kg}$; Roche Diagnostics, Sydney, Australia) 15 min before being placed in the test chambers on test day. Rats in the two SAL groups received equivalent injections of $0.9 \%$ saline.

To minimize responses to novelty on the test day, all rats received seven habituation sessions on consecutive days in which they were placed in the test chamber for $60 \mathrm{~min}$ with no collar present. On the last four of these days, all rats received a subcutaneous injection of saline $15 \mathrm{~min}$ before being placed in the chamber. This habituated them to the injection procedure before the test day.

On the test day, for the two CAT groups, pieces of a worn collar were attached to the three alligator clips in the arena. The two CONTROL groups were exposed to pieces of an unworn collar. The unworn cat collar had a faint odor detectable by a human, and presumably the rodent, so the control groups were therefore exposed to a novel nonpredatory odor. To minimize contamination between CAT and CONTROL groups, rats in the two CAT groups were either (1) run on the same test day but after CONTROL rats or (2) run on a different test day to CONTROL rats. A total of three separate test days were used in a staggered sequence. Rats in the CAT and CONTROL conditions were always tested in different test chambers, albeit of identical design and placed within the same room. Wherever possible, rats in the different groups were tested in a way that counterbalanced time of day across groups (Funk and Amir, 2000). All testing was conducted under dim red light conditions during the dark cycle of the rats.

During habituation and testing, rats were transported to and from the apparatus in light-excluding cardboard boxes with fresh sawdust bedding. They also remained in these boxes for the 15 min between drug injection and testing.

On the test day, rats remained in the test chamber for a total of $60 \mathrm{~min}$, after which they were taken to an adjacent room for perfusion.

\section{Immunohistochemistry}

All rats were deeply anesthetized with pentobarbitone $(120 \mathrm{mg} / \mathrm{kg}$, i.p.) and then perfused transcardially with $200 \mathrm{ml}$ of $0.1 \mathrm{M}$ PBS, followed by $400 \mathrm{ml}$ of $4 \%$ paraformaldehyde in PBS, $\mathrm{pH}$ 7.3. The brains were removed, blocked in the coronal plane, and placed in paraformaldehyde overnight at $4^{\circ} \mathrm{C}$. They were then placed in $15 \%$ sucrose for $24 \mathrm{hr}$, followed by $30 \%$ sucrose for $48 \mathrm{hr}$. After this, the tissue blocks were placed on microtome stages, frozen to $-17^{\circ} \mathrm{C}$, and sectioned at $40 \mu \mathrm{m}$. The entire brain was sectioned coronally apart from one of the olfactory bulbs for each rat, which were sectioned in the sagittal plane to allow clearer elucidation patterns of Fos expression in the accessory olfactory bulb (AOB). Consecutive sections were placed sequentially across four vials of $0.1 \mathrm{M}$ phosphate buffer (PB).

Free-floating sections were incubated for $30 \mathrm{~min}$ in $1 \%$ hydrogen peroxide in $\mathrm{PB}$ and then for $30 \mathrm{~min}$ in 3\% normal horse serum in $\mathrm{PB}$. The sections were then incubated in primary c-Fos antibody $(\mathrm{Ab})$ for $72 \mathrm{hr}$ at $4^{\circ} \mathrm{C}$ (rabbit polyclonal; reacts with c-Fos p62 of mouse, rat, and human; non-cross-reactive with FosB, Fra-1, or Fra-2; Santa Cruz Biotechnology, Santa Cruz, CA). The primary Ab was diluted 1:2000 in phosphatebuffered horse serum (PBH) $(0.1 \%$ bovine serum albumin, $0.2 \%$ Triton $\mathrm{X}-100$, and $2 \%$ normal horse serum in $\mathrm{PB}$ ). Sections were then washed for $30 \mathrm{~min}$ in $\mathrm{PB}$ at room temperature and incubated for $1 \mathrm{hr}$ at room temperature in secondary $\mathrm{Ab}$ (biotinylated anti-rabbit IgG made in goat; diluted 1:500 in PBH; Vector Laboratories, Burlingame, CA). They were then washed in $\mathrm{PB}$ for an additional $30 \mathrm{~min}$ and then incubated for $1.5 \mathrm{hr}$ in ExtrAvidin-horseradish peroxidase (diluted 1:1000 in PBH; Sigma, St. Louis, MO). After this, they were washed three more times (30 min) in $\mathrm{PB}$, after which horseradish peroxidase activity was visualized with nickel diaminobenzodine and glucose oxidase reaction as described previously (Dielenberg et al., 2001b). This reaction was terminated after $10 \mathrm{~min}$ by washing in $\mathrm{PB}$.

The sections were then mounted on subbed slides, dehydrated in ascending concentrations of ethanol, xylene cleared, and coverslipped.

\section{Counting of labeled cells}

The method described above produces a black oval-shaped immunoprecipitate confined to the cell nucleus of Fos-positive cells. This was quantified microscopically at 50 sites using a brain atlas for guidance (Paxinos and Watson, 1997). Only darkly labeled oval-shaped nuclei were counted. Three slightly different approaches to quantification were used relating to the following: (1) coronal sections for the entire brain; (2) sagittal sections from the accessory olfactory bulb; and (3) coronal sections from the main olfactory bulb (MOB).

Coronal sections. Coronal sections were viewed under either a $20 \times$ or $40 \times$ objective, and an optical graticule was used to manually quantify the number of Fos-positive neurons in each region. The numbers of positive nuclei that fell within a $0.5 \times 0.5 \mathrm{~mm}$ area $(20 \times$ objective $)$ or $0.25 \times 0.25$ area $(40 \times$ objective) in each region of interest were counted from one section per rat by an observer who was blind to group assignment. The regions quantified are shown in Figure 1, and counts are reported in Table 1. 
In several cases, the designated area to be counted was substantially larger than the boundaries of the graticule (Fig. 1). In such cases, the graticule was placed in a fixed position within the region of interest relative to known anatomical landmarks. In other cases, the designated area was smaller than the boundaries of the graticule. In such cases, only the region of interest, not extraneous areas, were counted.

Accessory olfactory bulb. The AOB was quantified microscopically ( $40 \times$ objective) in sagittal sections. The graticule was used to quantify Fos-positive cells in 12 subregions within the anterior and posterior glomerular, mitral, and granule cell layers. Each region quantified constituted a $0.25 \times 0.25 \mathrm{~mm}$ square, as shown in Figure $2 e$, by an observer who was blind to group assignment. For statistical analysis, counts from adjacent regions were summed (Fig. 2f) to provide a total of six counts per rat for the AOB. For comparison purposes, an additional count from the mitral cell layer was made from coronal sections (Fig. 1), the data for which are presented in Table 1.

Main olfactory bulb. For the MOB, a number (one to five) of representative coronal sections from each rat at a level of $\sim 6.7 \mathrm{~mm}$ anterior to bregma were obtained. Fos expression in the entire glomerular layer of the MOB was counted microscopically for each of these sections by an observer who was blind to group assignment. For the purposes of analysis, the glomerular layer was subdivided into six separate sectors corresponding to the dorsomedial, dorsolateral, medial, lateral, ventromedial, and ventrolateral areas (Fig. 3e). A Fos count for each of these sectors was obtained for each rat by averaging counts for each sector from all the different sections counted.

For comparison purposes, two additional counts were made in the MOB in the granule cell layer using the approach described above for coronal sections. The regions counted are shown in Figure 1, and the data are presented in Table 1.

\section{Preparation of images}

Digital images were made of representative pieces of tissue for illustrating the distribution of Fos-positive cells in key areas. The digitized images were produced with a Micropublisher 3.3 megapixel camera attached to an Olympus Optical (Tokyo, Japan) BX40 light microscope. Images were acquired using a G4 dualprocessor Macintosh computer (Apple Computers, Cupertino, CA) running Adobe Photoshop 7.0 (Adobe Systems, San Jose, CA). The only post-production enhancement was conversion of color images to black and white and the adjustment of brightness for printing purposes.

\section{Behavioral analysis}

The videotapes acquired during testing were scored using the ODLog scoring program (Macropod Software, Armidale, Australia). Scoring was accomplished by an observer who was blind to group assignment. Because of the confined conditions within the test chamber, only a very limited behavioral repertoire was evident in the rats. Initial examination of videotape recordings indicated that the following behaviors were regularly observed, scored as follows: (1) contact, defined as the rat making contact with the collar with either the nose or mouth; (2) grooming, defined as the rat preening or licking itself in a typical manner; and (3)
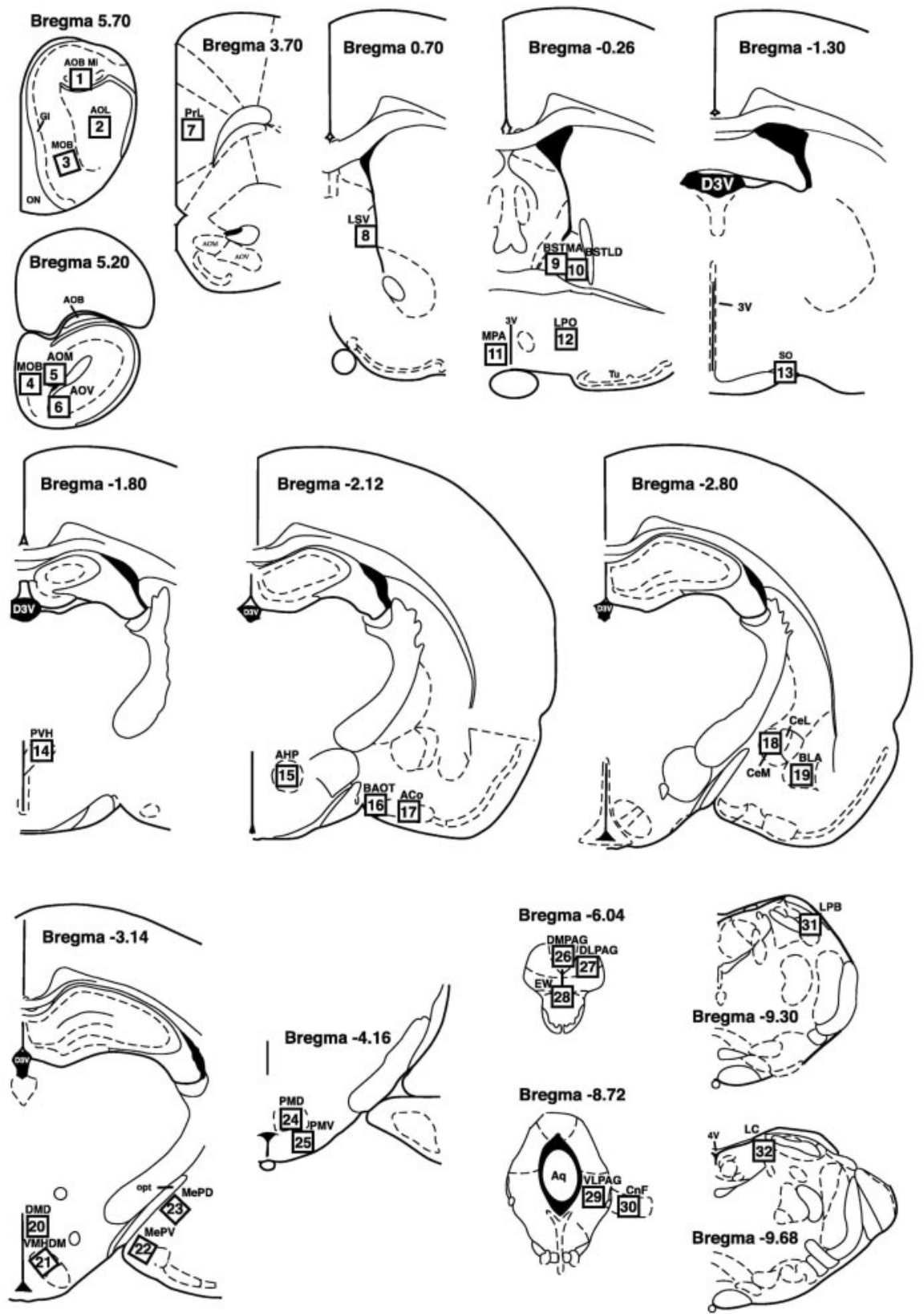

Figure 1. Schematic diagram of brain regions counted in coronal sections. The figure is adapted from the atlas of Paxinos and Watson (1997) and shows the 32 regions in which Fos immunoreactivity was quantified in the coronal plane and reported in Table 1. Open squares indicate the approximate positions of the grid within which the Fos counts were made. For counts of Fosimmunoreactive cells corresponding to this diagram and abbreviations, see Table 1.

rearing, defined as the rat standing on both hind legs, a behavior that situated the head of the rat well above the level of the collar stimulus. Freezing was not scored because it was not easily discerned from immobility in the videotapes of the test sessions attributable to the ceiling position of the video cameras. In any case, our previous research has suggested minimal freezing to a worn cat collar stimulus even when presented under confined conditions (McGregor et al., 2002), and this also appeared to be the case in the present study.

\section{Data analysis}

One-way ANOVA was used to compare the four groups on the scored behaviors and the number of Fos-positive nuclei in the brain regions counted. Significant ANOVAs were followed by a post hoc test (Newman-Keuls) to enable specific group comparisons. Levene's test was used to test for homogeneity of variance, and, when this requirement was not 
Table 1. Counts of Fos-positive cells from coronal brain sections

\begin{tabular}{|c|c|c|c|c|c|}
\hline Region & Reference (Fig.1) & CONTROL-SAL $(n=6)$ & CONTROL-MDZ $(n=4)$ & CAT-SAL $(n=7)$ & CAT-MDZ $(n=6)$ \\
\hline \multicolumn{6}{|c|}{ Sites at which midazolam reduces cat odor-induced c-fos expression } \\
\hline AON (lateral) & 2 & $16(6.8)$ & $2(2)$ & $31(7.9)^{b, c}$ & $10.5(2.5)$ \\
\hline AON (medial) & 5 & $18.2(4.2)$ & $9(3.5)$ & $35.4(5.2)^{a, b, c}$ & $12(2.2)$ \\
\hline AON (ventral) & 6 & $21(7.3)^{b, c}$ & $2.3(0.8)$ & $37.9(5.0)^{a, b, c}$ & $5.2(1.0)$ \\
\hline MPC (prelimbic) & 7 & $18(3.9)$ & $13(5.0)$ & $60.7(7.1)^{a, b, c}$ & $18.5(0.9)$ \\
\hline Lateral septum (ventral) & 8 & $15(3.14)$ & $6(2.7)$ & $100.7(9.8)^{a, b, c}$ & $19.5(3.7)$ \\
\hline BNST (medial anterior) & 9 & $7.8(1.8)$ & $2.7(0.9)$ & $17.1(1.8)^{a, b, c}$ & $7.7(1.0)$ \\
\hline Medial preoptic area & 11 & $6.5(1.8)$ & $4.7(1.6)$ & $17(3.1)^{a, b, c}$ & $6(1.6)$ \\
\hline Lateral preoptic area & 12 & $4.7(1.7)$ & $2.3(0.5)$ & $24.7(3.5)^{a, b, c}$ & $5.7(2.0)$ \\
\hline Anterior hypothalamic area (posterior) & 15 & $20(4.1)$ & $12.8(4.3)$ & $63.7(6.5)^{a, b, c}$ & $28(6.1)$ \\
\hline Dorsomedial hypothalamus & 20 & $12.2(1.8)$ & $5.8(2.2)$ & $34.2(6.6)^{a, b, c}$ & $18.7(2.8)$ \\
\hline MePV & 22 & $12(4.2)$ & $6(1.0)$ & $65.4(6.2)^{a, b, c}$ & $47.5(7.3)^{a, b}$ \\
\hline PMd & 24 & $6(1.6)$ & $1.8(1.1)$ & $63.9(9.9)^{a, b, c}$ & $24.8(9.3)^{a, b}$ \\
\hline \multicolumn{6}{|c|}{ Sites at which midazolam does not significantly affect cat odor-induced c-fos expression } \\
\hline AOB (mitral layer) & 1 & $18.5(2.6)$ & $17.3(3.5)$ & $44.4(6.5)^{a, b}$ & $50.3(3.6)^{a, b}$ \\
\hline PVN & 14 & $7.7(1.4)$ & $15.5(5.1)$ & $51.7(14.4)^{a, b}$ & $68.2(14.7)^{a, b}$ \\
\hline $\mathrm{BAOT}(40 \times)$ & 16 & $5.8(2.1)$ & $2.3(1.0)$ & $17(2.5)^{a, b}$ & $12.5(1.6)^{b}$ \\
\hline VMH (dorsomedial) $(40 \times)$ & 21 & $3(1.0)$ & $6.3(6.3)$ & $36.9(6.6)^{a, b}$ & $28.5(8.1)^{a, b}$ \\
\hline PAG (dorsomedial) & 26 & $5.7(1.0)$ & $7.3(1.7)$ & $30.9(5.0)^{a, b}$ & $25.8(3.6)^{a, b}$ \\
\hline PAG (dorsolateral) & 27 & $7.2(1.4)$ & $6.5(2.3)$ & $35.9(4.3)^{a, b}$ & $31.3(7.0)^{a, b}$ \\
\hline PAG (ventrolateral) & 29 & $5.2(1.4)$ & $5.3(1.4)$ & $38.3(11.1)^{a, b}$ & $29.2(6.7)^{a, b}$ \\
\hline Cuneiform nucleus & 30 & $4.5(1.9)$ & $11.7(1.9)$ & $34.6(6.7)^{a, b}$ & $25(4.6)^{a, b}$ \\
\hline Locus ceruleus & 32 & $0.7(0.5)$ & $0.5(0.9)$ & $8.9(3.2)^{a, b}$ & $15.7(3.2)^{a, b}$ \\
\hline \multicolumn{6}{|c|}{ Sites at which midazolam increases c-fos expression } \\
\hline BNST (dorsolateral) $(40 \times)$ & 10 & $5.2(1.6)$ & $14.5(3.5)^{a}$ & $9.3(2.5)$ & $36(6.6)^{a, b, d}$ \\
\hline CEA & 18 & $4.2(1.0)$ & $20.8(4.0)^{a, d}$ & $2.9(1.2)$ & $32(7.1)^{a, d}$ \\
\hline Edinger-Westphal nucleus (40×) & 28 & $3.17(1.2)$ & $14.5(5.1)^{a}$ & $7(1.7)$ & $21.7(4 .)^{a, d}$ \\
\hline Lateral parabrachial nucleus & 31 & $2.7(1.0)$ & $7.8(2.7)^{a}$ & $3.9(0.9)$ & $23.6(5.2)^{a, b, d}$ \\
\hline \multicolumn{6}{|c|}{ Sites at which midazolam reduces basal c-fos expression } \\
\hline $\mathrm{MOB}(+5.7)(40 \times)$ & 3 & $36.7(11.4)^{b, c}$ & $7(2.6)$ & $38.7(7.7)^{b, c}$ & $10.6(1.6)$ \\
\hline $\mathrm{MOB}(+5.2)(40 \times)$ & 4 & $25.7(6.6)^{b, c}$ & $6.3(1.4)$ & $38.9(5.5)^{b, c}$ & $9.3(1.8)$ \\
\hline \multicolumn{6}{|l|}{ Sites that were mostly unaffected } \\
\hline Supraoptic nucleus $(40 \times)$ & 13 & $2.2(0.8)$ & $1.3(0.9)$ & $5(2.0)$ & $4.8(1.6)$ \\
\hline Anterior cortical amygdala (40×) & 17 & $12.2(2.2)$ & $5.3(2.0)$ & $16.3(1.6)$ & $11.5(1.9)$ \\
\hline Basolateral amygdala & 19 & $5.7(1.8)$ & $1.3(1.0)$ & $11.6(3.0)^{b}$ & $6.8(1.7)$ \\
\hline Medial amygdala (posterodorsal) & 23 & $9.3(1.9)$ & $3.5(0.6)$ & $11.6(3.2)$ & $5(1.0)$ \\
\hline Premammillary nucleus (ventral) & 25 & $12.5(3.3)$ & $2.8(1.5)$ & $8.6(3.2)$ & $4.2(1.2)$ \\
\hline
\end{tabular}

Data are mean (SEM). All counts were made within graticule at $20 \times$ magnification, except when indicated in which $40 \times$ magnification was used (40 $\times$ ). PVN, Paraventricular nucleus; BA0T, bed nucleus of the accessory olfactory tract. ${ }^{a} p<0.05$ versus CONTROL-SAL group; ${ }^{b} p<0.05$ versus CONTROL-MDZ group; ${ }^{c} p<0.05$ versus CAT-MDZ group; ${ }^{d} p<0.05$ versus CAT-SAL group.

satisfied, data were $\log 10(x+1)$ transformed before analysis. A probability level of 0.05 was used to determine statistically significant differences between groups.

\section{Results}

\section{Behavior}

The behavioral results are shown in Figure 4. Relative to controls, rats exposed to cat odor with vehicle pretreatment (group CATSAL) showed less stimulus contact, less grooming, and higher levels of rearing than rats exposed to an unworn collar with vehicle pretreatment (group CONTROL-SAL). Rearing was very prevalent in cat odor-exposed rats and maximized distance from the odor stimulus. These rats also frequently pushed their noses through the spaces between the metal bars forming the grid floors. Occasional jumping was seen as some cat odor-exposed rats attempted to escape the test chamber. Cat odor-exposed rats were notably reactive to handling when being removed from the chamber.

When comparing groups CAT-SAL and CAT-MDZ, it is evident that midazolam significantly attenuated the effects of cat odor on stimulus contact and rearing (Fig. 4). Rats in the CATMDZ group were often seen to chew the worn collars. In com- paring groups CONTROL-SAL and CONTROL-MDZ, it is evident that midazolam significantly reduced grooming and rearing in rats exposed to an unworn collar. Midazolam-treated cat odorexposed rats were less reactive to handling when being removed from the test chamber and showed notably less muscle tension but were not frankly ataxic.

\section{Overview of regional Fos expression}

The overall pattern of Fos expression seen in cat odor-exposed rats (Table 1; Figs. 2-7) was comparable with our previous study, in which rats were allowed to retreat and hide from the cat odor stimulus (Dielenberg et al., 2001b). Thus, in the present study, the group CAT-SAL showed pronounced Fos expression relative to group CONTROL-SAL in the medial (but not the central) amygdala, BNST, the lateral septum, the dorsal premammillary nucleus of the hypothalamus (PMd), the ventromedial hypothalamic nucleus, the lateral and medial preoptic areas, the anterior hypothalamic area, the three major subregions of the periaqueductal gray, and the cuneiform nucleus. Novel findings pertained to the effects of midazolam in these and other brain regions and 

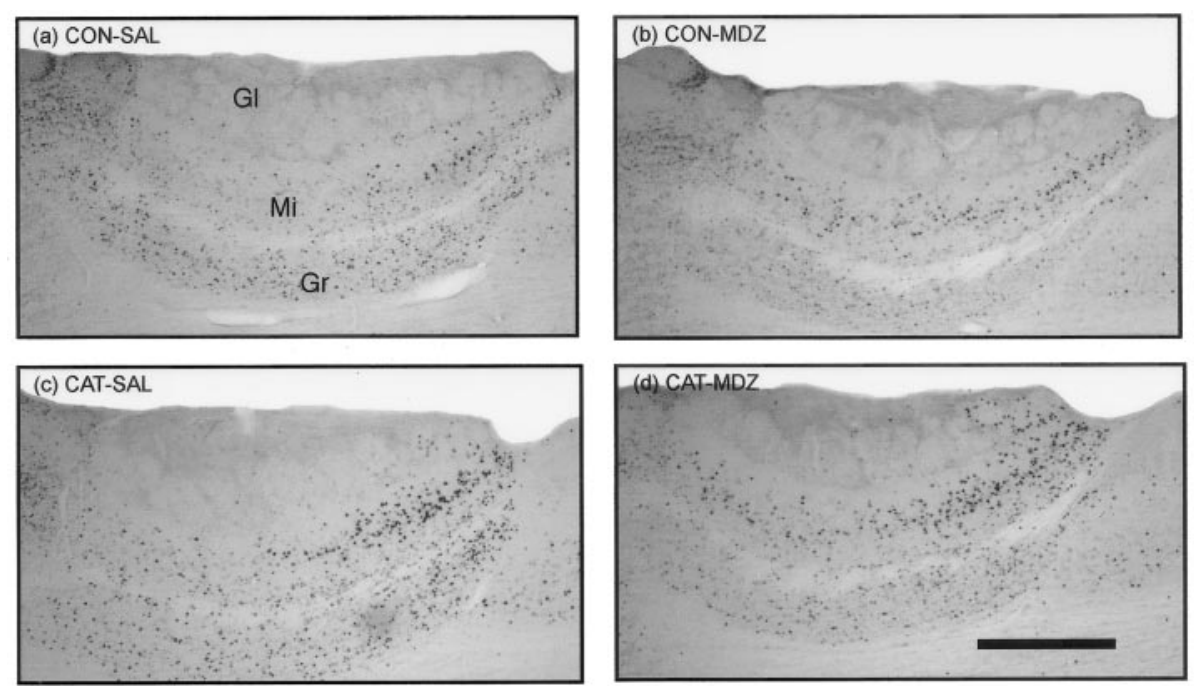

(e)
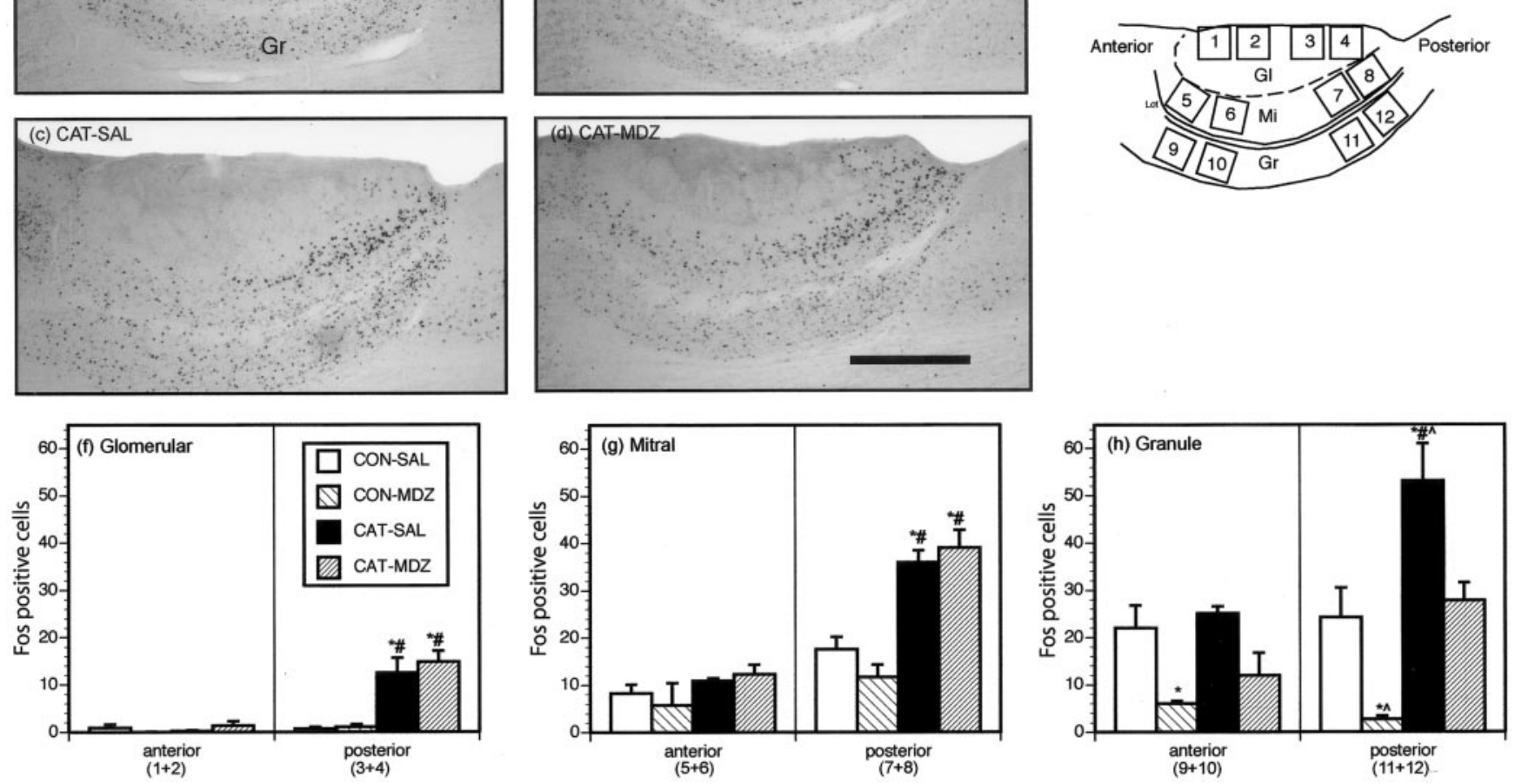

Figure 2. Results from the AOB. $a, b, c$, and $d$ show photomicrographs of Fos expression in representative animals from groups CON-SAL, CON-MDZ, CAT-SAL, and CAT-MDZ, respectively. Scale bar, $200 \mu \mathrm{m}$. e shows a schematic diagram of a sagittal section of the AOB showing the 12 subregions in which Fos immunoreactivity was quantified. Open squares indicate the position of the $0.25 \times$ $0.25 \mathrm{~mm}$ grid within which the Fos counts were made. $f, g$, and $h$ show Fos counts for the regions in the four experimental groups in the glomerular (GI), mitral (Mi), and granule (Gr) cell layers, respectively. ${ }^{*} p<0.05$, significantly different from CONTROL-SAL group; \#p $<0.05$, significantly different from CONTROL-MDZ group; ${ }^{\wedge} p<0.05$, significantly different from CAT-MDZ group; Newman-Keuls post hoc tests.

the effects of cat odor exposure on Fos expression in the accessory and main olfactory bulb.

\section{The accessory olfactory bulb}

The $\mathrm{AOB}$ is the primary projection area of the vomeronasal organ and is primarily implicated in the processing of pheromones. It has been subdivided on functional and anatomical grounds into an anterior and posterior part, with each having projections from distinct cell types in the vomeronasal sensory epithelium expressing different types of the G-proteins (Halpern and MartinezMarcos, 2003). The anterior AOB may be primarily activated by pheromones related to reproductive behavior, whereas the posterior AOB processes cues relating to inter-male territorial marking and aggression (Kumar et al., 1999).

Fos counts for the $\mathrm{AOB}$ and representative photomicrographs from this region are displayed in Figure 2. Rats exposed to cat odor (CAT-SAL group) displayed increased Fos expression in the glomerular, mitral, and granule cell layers of the posterior $\mathrm{AOB}$ relative to controls (CONTROL-SAL), whereas counts in the anterior AOB did not differ between these two groups. The observation that cat odor activates the posterior AOB invites the interesting suggestion that this stimulus is processed in a "pheromone-like" manner.

Midazolam did not affect cat odor-induced Fos expression in the glomerular or mitral cell layers of the AOB (group CAT-MDZ vs group CAT-SAL). However, midazolam significantly attenu- ated basal Fos expression in the granule cell layer of the anterior $\mathrm{AOB}$, as well as basal and cat odor-induced Fos expression in this layer in the posterior $\mathrm{AOB}$ (Fig. 2). The granule cell layer of the AOB is strongly GABAergic (Quaglino et al., 1999) and contains GABA subunit configurations that bind benzodiazepines (Laurie et al., 1992; Fritschy and Mohler, 1995). These observations correspond well with the inhibitory effects of midazolam in this AOB layer.

\section{The main olfactory bulb and anterior olfactory nuclei}

As is typically seen in olfactory studies (Sallaz and Jourdan, 1993; Klintsova et al., 1995; Guthrie and Gall, 2003), neurons in the glomerular layer of the MOB expressed Fos in a circular pattern surrounding the glomerular neuropil (Fig. 3). Studies using 2-DG autoradiography (Sallaz and Jourdan, 1993; Johnson et al., 2002), visualization of c-fos mRNA (Guthrie and Gall, 2003), or Fos immunohistochemistry (Sallaz and Jourdan, 1993; Klintsova et al., 1995) demonstrate that specific odorants activate a unique topography of glomeruli within the MOB. Here we undertook a simple analysis of MOB activation to cat odor by counting Fos in six different sectors of the glomerular layer at a level approximately midway along the anteroposterior extent of the bulb.

Results are presented in Figure 3. In contrast to the AOB, high basal levels of Fos expression were evident in the MOB (group CON-SAL), perhaps reflecting a range of olfactory stimuli present in the laboratory, home cages, and test chambers that the present experiment did not attempt to control. Some topography 

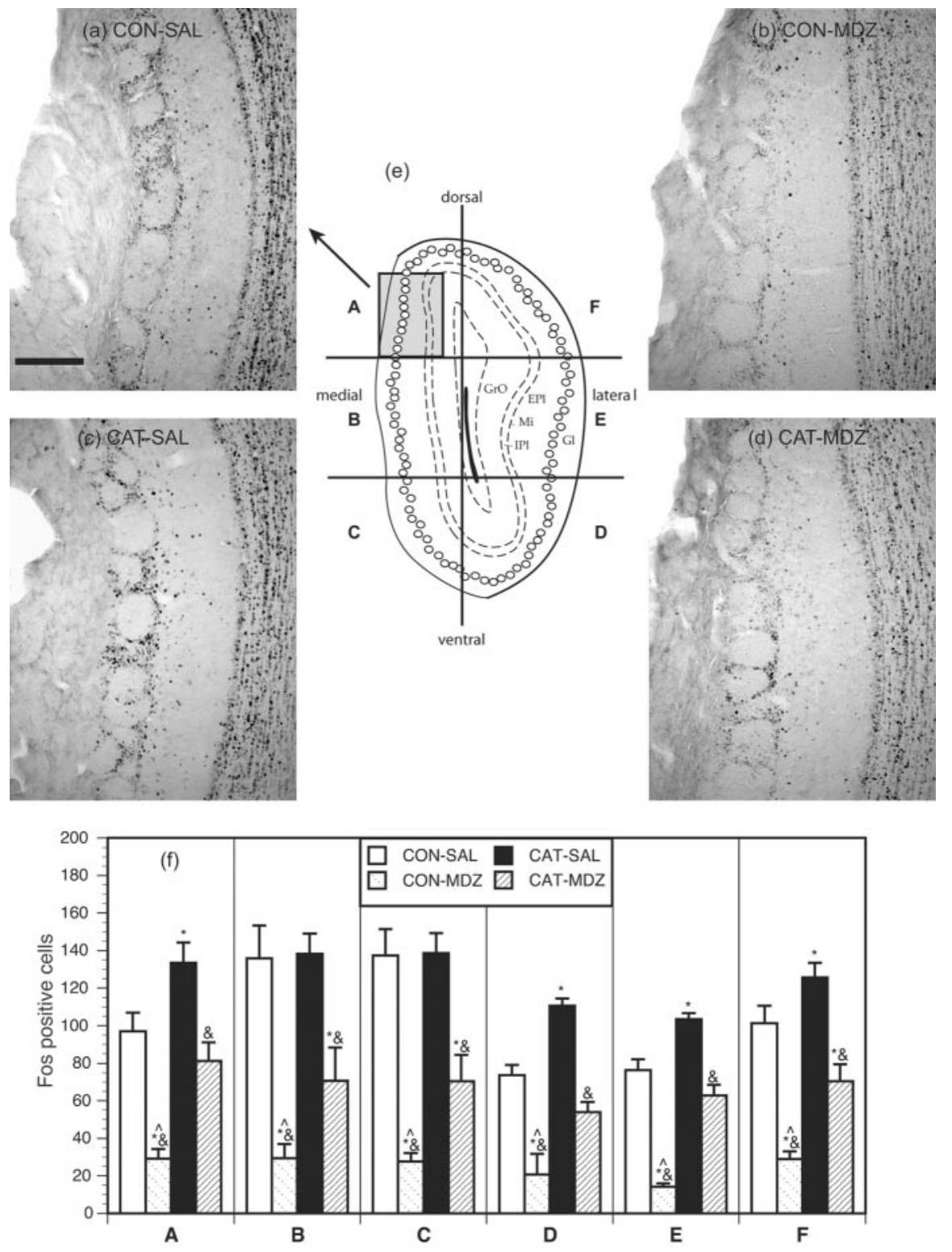

Figure 3. Results from the MOB. $a, b, c$, and $d$ show photomicrographs of Fos expression in sector $A$ of the MOB from representative animals from groups CON-SAL, CON-MDZ, CAT-SAL, and CAT-MDZ, respectively. Scale bar, $200 \mu \mathrm{m} . e$, Schematic diagram, adapted from Paxinos and Watson (1997), showing a coronal section of the MOB indicating approximate position of glomeruli (open circles) and indicating the six sectors (A-F) in which Fos immunoreactivity in the glomerular layer was quantified. The area represented in photomicrographs $a-d$ is shown by the gray square in sector $A$. $f$, The counts for sectors $A-F$ in the four experimental groups. ${ }^{*} p<0.05$, significantly different from CONTROL-SAL group; ${ }^{\&} p<0.05$, significantly different from CAT-SAL group; ${ }^{\wedge} p<0.05$, significantly different from CAT-MDZ group; Newman-Keuls post hoc tests. EPI, External plexiform layer; IPI, internal plexiform layer; $\mathrm{Gl}$, glomerular layer; $\mathrm{GrO}$, granule layer.

was evident in this basal Fos expression with highest levels of activation seen in the medial and ventromedial sectors of the glomerular layer (Fig. 3, CON-SAL).

Cat odor-exposed rats (CAT-SAL) displayed a small but significant increase in Fos expression relative to controls (CONTROL-SAL) in four of the six sectors quantified within the glomerular layer (Fig. 3). There was also a tendency toward higher Fos expression in the CAT-SAL group in the two areas of the granule cell layer that were quantified (Table 1).

Interestingly, midazolam had a powerful effect in the MOB, reducing the high basal level of Fos in all of the glomerular and granule cell regions that were counted (Fig. 3; Table 1, compare groups CONTROL-SAL, CONTROL-MDZ). Midazolam also re- duced MOB Fos expression in cat odorexposed rats (compare groups CAT-SAL, CAT-MDZ). The inhibitory effect of midazolam was easily seen in the glomerular layer (Fig. 3), with the number of glomeruli circled by Fos staining greatly reduced in midazolam-treated rats.

This to our knowledge is the first demonstration of an inhibitory effect of benzodiazepines on basal and odor-stimulated Fos expression in the $\mathrm{MOB}$ and suggests the intriguing possibility that humans treated with benzodiazepines may exhibit alterations in olfactory function. There is well documented GABAergic circuitry and benzodiazepine binding sites in the MOB (Laurie et al., 1992; Fritschy and Mohler, 1995), agreeing well with the midazolam effect observed in this region.

Cat odor exposure also increased Fos expression in the three subdivisions of the anterior olfactory nucleus (AON), and this was also significantly reduced by $\mathrm{mi}$ dazolam (Table 1). As with the MOB, midazolam decreased basal Fos expression in the AON in control rats, suggesting a general inhibitory effect of the drug on Fos expression in this region. The AON receives the majority of its input from the $\mathrm{MOB}$ and little if any from the AOB (Shipley et al., 1995), and it is not surprising then that group differences in the pattern of Fos expression seen here should mirror that seen in the MOB.

\section{Medial prefrontal cortex}

Exposure to cat odor caused a striking activation of Fos expression in the prelimbic region of the medial prefrontal cortex (MPC) (Table 1). Several other frontal cortical regions showed evidence of activation in cat odor-exposed rats, including the infralimbic and lateral orbital regions (data not shown). Activation of prelimbic cortex was not evident in our previous study in which rats were given the opportunity to hide from the cat odor stimulus (Dielenberg et al., 2001b). This suggests the possibility that the MPC becomes active in situations in which predator cues are inescapable, agreeing with previous suggestions that the MPC is involved in coping responses during inescapable stress (Espejo, 1997; Berridge et al., 1999; Keay and Bandler, 2001).

Midazolam dramatically altered Fos expression in the prelimbic cortex, with cat odor effects completely absent in midazolam pretreated rats (Table 1, compare groups CAT-SAL, CAT-MDZ). Midazolam had no effects on basal Fos expression within this region, suggesting a specific effect of the drug on anxietyrelated activation.

\section{The lateral septum}

The pattern of results in the lateral septum (ventral part) was similar to that seen in the MPC: a strongly activation by cat odor 


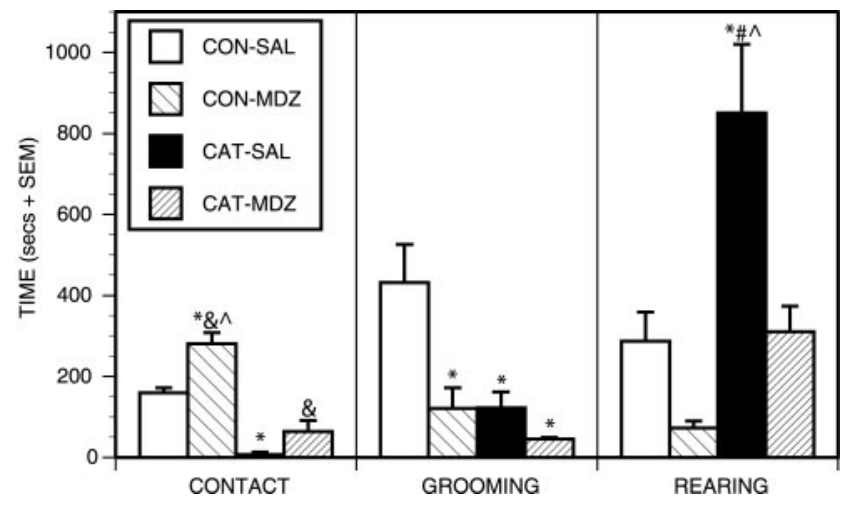

Figure 4. Behavioral results. The duration of stimulus contact, grooming, and rearing in each of the four groups during the 60 min test period. ${ }^{*} p<0.05$, significantly different from CONTROL-SAL group; $\# p<0.05$, significantly different from CONTROL-MDZ group; ${ }^{*} p<0.05$, significantly different from CAT-SAL group, ${ }^{\wedge} p<0.05$, significantly different from CAT-MDZ group; Newman-Keuls post hoc tests.
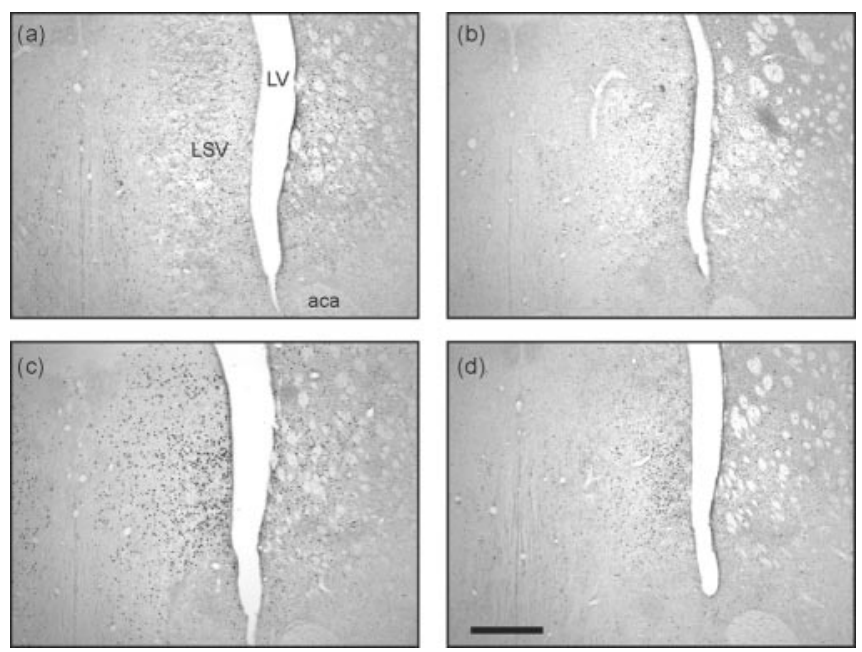

Figure 5. Photomicrographs of the lateral septum. Taken from coronal sections located $\sim 0.70 \mathrm{~mm}$ anterior to bregma showing Fos expression in representative rats from groups CONTROL-SAL ( $a$ ), CONTROL-MDZ ( $b$ ), CAT-SAL ( $c$ ), and CAT-MDZ ( $d$ ). Note the high levels of Fos expression produced by cat odor exposure in c and the reduction in produced by midazolam evident when comparing c and $d$. Scale bar, $500 \mu \mathrm{m}$. For location of brain region corresponding to these photomicrographs, see Figure 1, region 8. LSV, Lateral septum (ventral part); LV, lateral ventricle; aca, anterior commisure.

that was almost completely reversed by midazolam (Fig. 5). There was little cat odor-related Fos expression in other septal regions, including the diagonal band of Broca (Mongeau et al., 2003).

The septum is suggested to be a key mediator of anxiety states and a principle site of action for anti-anxiety drugs (Gray and McNaughton, 2000). Benzodiazepines injected directly into this region are anxiolytic across a range of animal models (Menard and Treit, 1999), and the lateral septum (ventral part) has been identified as a key region that differentiates active versus passive forms of defense (Mongeau et al., 2003). This area was strongly activated in mice showing freezing rather than fleeing to an anxiogenic ultrasonic stimulus (Mongeau et al., 2003). It might then be hypothesized that the ability of benzodiazepines to inhibit freezing and cause increased approach toward predator cues (Dielenberg et al., 1999; McGregor and Dielenberg, 1999; McGregor et al., 2002) may be coordinated from this region.
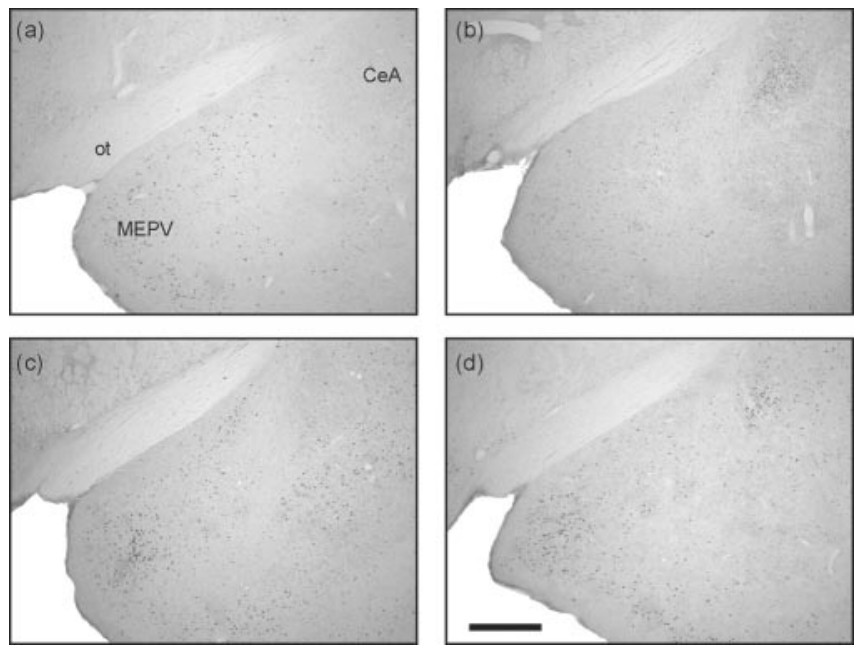

Figure 6. Photomicrographs of the amygdala. Taken from coronal sections located $\sim 3.14$ $\mathrm{mm}$ posterior to bregma showing Fos expression in the amygdala of representative animals from groups CONTROL-SAL ( $a$ ), CONTROL-MDZ ( $b$ ), CAT-SAL ( $c$ ), and CAT-MDZ ( $d$ ). Note that cat odor increased Fos expression in the medial amygdala ( $c$ ) and that this was only weakly affected by midazolam ( $d$ ). Midazolam itself induced Fos expression in the central nucleus ( $b$, d). Scale bar, $500 \mu \mathrm{m}$. For location of brain region corresponding to these photomicrographs, see Figure 1, regions 22 and 23. ot, Optic tract; MEPV, medial amygdala (posteroventral); CeA, central nucleus of the amygdala.
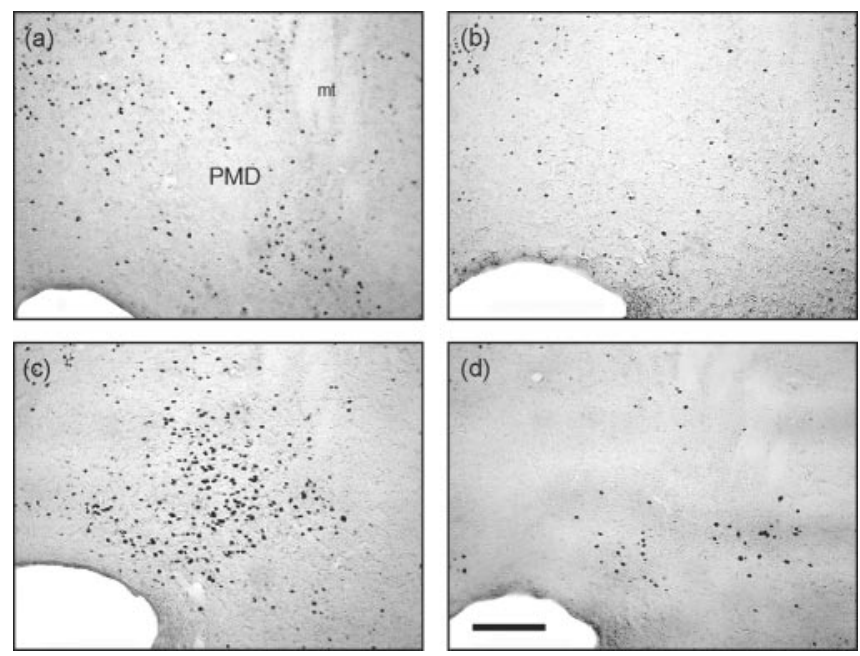

Figure 7. Photomicrographs of the dorsal premammillary nucleus. Taken from coronal sections located $\sim 4.16 \mathrm{~mm}$ posterior to bregma showing Fos-immunoreactive cells from representative rats from groups CONTROL-SAL (a), CONTROL-MDZ (b), CAT-SAL (c), and CAT-MDZ (d). Note the profound inhibition of Fos expression produced by midazolam evident when comparing $c$ and $d$. Scale bar, $200 \mu \mathrm{m}$. For location of brain region corresponding to these photomicrographs, see Figure 1, region 24. mt, Mamillothalamic tract; PMD, dorsal premammillary nucleus.

\section{The extended amygdala}

Primary projections from the AOB terminate in the medial nucleus of the amygdala and BNST (Scalia and Winans, 1975). As reported previously (Dielenberg et al., 2001b), cat odor elicited a pronounced activation of the posteroventral part of the medial amygdala (MePV) (Fig. 6), suggesting that this may reflect a downstream effect resulting from AOB activation. Midazolam caused a modest reduction in cat odor-induced Fos expression in the MePV. This could reflect the upstream effect of midazolam in the granule cell layer of the $\mathrm{AOB}$ or a direct effect within the medial amygdala, in which a modest density of benzodiazepine binding sites is evident (Kaufmann et al., 2003). 
A very recent report indicates that excitotoxic lesions of the medial amygdala disrupt the defensive response to cat odor in rats but have no effect on shock-motivated defensive freezing ( $\mathrm{Li}$ et al., 2004). Whether this reflects a sensory deficit (i.e., inability to process pheromonal information) or a fundamental interruption of emotion-related circuitry (Dayas et al., 1999) remains to be determined.

Cat odor exposure also caused a modest increase in Fos expression in the anterior part of the medial region of the BNST, with this effect reversed by midazolam. The BNST receives a direct projection from the AOB (Scalia and Winans, 1975) and also has intimate connections with the medial amygdala, of which it is considered to be a rostral extension of the medial nucleus of the amygdala and has extensive projections from this region. The BNST has been implicated in the response of rats to the putative predator odor trimethylthiazoline, with lesions in this region preventing freezing to this stimulus (Fendt et al., 2003). Other emerging evidence suggest a fundamental role of the BNST in mediating unconditioned fear responses to a range of threatrelated stimuli (Lee and Davis, 1997; Walker et al., 2003).

Interestingly, midazolam by itself increased Fos expression in both the central nucleus of the amygdala (CEA) and the dorsal part of the lateral BNST. The CEA contains a high density of benzodiazepine binding sites (Kaufmann et al., 2003) and is stimulated by a wide range of anxiogenic, anxiolytic, and reinforcing drugs (Stephenson et al., 1999; Allen et al., 2003; Singewald et al., 2003). Benzodiazepine induction of c-fos expression in the CEA was evident in one previous report (Beck and Fibiger, 1995). The lateral BNST is considered to be a rostral extension of the CEA and displays similar activation to various pharmacological challenges (Allen et al., 2003).

It is notable that the patterns of Fos expression observed suggest that the lateral BNST and the CEA were not in the least activated by an innately anxiogenic stimulus (cat odor) yet showed strong activation to an anxiolytic drug (midazolam). This challenges any simplistic notion that Fos expression in the CEA and BNST can be simply equated with anxiety. It remains possible of course that these two regions are primarily inhibited rather than activated by fear-related stimuli, such as cat odor, with the Fos methodology unable to detect this inhibitory effect.

\section{The hypothalamus}

A variety of hypothalamic regions showed Fos expression as a result of cat odor exposure (Table 1). These include the lateral and medial preoptic areas, the anterior hypothalamic area, the PMd (Fig. 7), the ventromedial nucleus, the dorsomedial nucleus, and the paraventricular nucleus. Cat odor-induced Fos expression in most of these regions was significantly reduced by midazolam, with the exception of the ventromedial and paraventricular hypothalamic nuclei. The effect in the PMd was particularly interesting, with a large increase in Fos expression that was partly, although not entirely, prevented by midazolam pretreatment. There was no significant effect of cat odor exposure or midazolam in the ventral premammillary nucleus or the supraoptic nucleus (Table 1; Fig. 7).

The PMd is thought to act in concert with anterior hypothalamic regions and the dorsomedial part of the ventromedial hypothalamus (VMH) to form a "medial hypothalamic circuit" that integrates innate defensive responses to environmental threats (Canteras, 2002). Rats with PMd lesions fail to display speciestypical defensive responses to cats or cat odor (Canteras et al., 1997; Blanchard et al., 2003), whereas predators, predator odors, and other threat-related stimuli cause pronounced Fos expres- sion in this region (Canteras et al., 1997; Dielenberg et al., 2001b; Figueiredo et al., 2003; Mongeau et al., 2003). Defensive behavior is strongly modulated by GABAergic drugs, including midazolam, injected into medial hypothalamic regions (Milani and Graeff, 1987), inviting the hypothesis that this circuitry is a critical locus for benzodiazepine effects on defensive behavior.

Principal inputs to the medial hypothalamic circuit include forebrain and limbic regions activated here by cat odor and modulated by midazolam, including the prelimbic cortex, lateral septum, BNST, and MePV (Canteras, 2002). Thus, the inhibitory effect of midazolam treatment on PMd activation could reflect a dampening down of such inputs, as well as a local effect of the drug. The anterior hypothalamic region appears to gate septohippocampal input into the medial hypothalamic circuit, and the strong inhibitory action of midazolam in the anterior hypothalamus may be reflective of the powerful effect of the drug on upstream lateral septal regions.

In contrast, activation in the ventromedial hypothalamic part of the medial hypothalamic circuit was barely affected by midazolam. The VMH appears to integrate medial amygdala inputs into the circuit (Canteras, 2002), so the relatively weak action of midazolam in the medial amygdala and other accessory olfactory regions is consistent with this absence of a midazolam effect in the $\mathrm{VMH}$.

\section{Periaqueductal gray}

The major output of the medial hypothalamic defensive circuit is to the PAG, which is often considered as the lowest level of the defensive behavior hierarchy, involved in the execution of defensive autonomic and behavioral responses. Within the PAG, it has been hypothesized that the dorsal regions mediate active behavioral coping patterns, whereas the ventrolateral region triggers passive, quiescent responses to uncontrollable anxiogenic and painful stimuli (Keay and Bandler, 2001).

As reported previously (Canteras and Goto, 1999; Dielenberg et al., 2001b), cat odor increased Fos expression in the dorsomedial, dorsolateral, and ventrolateral PAG. Somewhat surprisingly, this PAG activation was not significantly affected by midazolam. Benzodiazepines injected directly into the PAG have apparent anxiolytic effects in the plus maze (Russo et al., 1993), and, from this, we predicted that midazolam would subdue anxiety-related Fos expression in this region. However, this was not the case. Some authors have questioned whether the effects of benzodiazepines in the PAG are specific to this site or are perhaps attributable to spreading of injections into the nearby dorsal raphe nucleus (Menard and Treit, 1999).

\section{Other brainstem regions}

Cat odor exposure induced modest Fos expression in the locus ceruleus, which, if anything, midazolam tended to amplify rather than reduce. Midazolam by itself increased Fos expression in the lateral parabrachial nucleus, with cat odor having no effect in this region. The lateral parabrachial nucleus is a major gustatory relay and is a site in which benzodiazepines exert a positive effect on food palatability (Higgs and Cooper, 1996). The increased contact with the cat collar stimulus seen in control rats (Fig. 3) was often associated with chewing of the collar stimulus, and this may be linked to midazolam effects in this region.

Midazolam also increased Fos expression in the EdingerWestphal nucleus, again a region in which cat odor had no effect. This region exhibits Fos expression to alcohol consumption and is a major locus of urocortin-sensitive pathways in the brain (Bachtell et al., 2003). Given the commonalities in the mode of 
action of alcohol and benzodiazepines, it is perhaps not surprising to note an effect of midazolam here.

\section{Discussion}

The present study involves a relatively novel approach to investigating the neuropharmacology of defensive behavior. Although many previous investigations have used Fos immunohistochemistry to investigate patterns of neural activation to either anxiogenic stimuli or psychoactive drugs, there are very few that have probed the combination of the two (Beck and Fibiger, 1995). This approach has the capacity to elucidate key neural effects that underpin anxiolytic drug actions and can generate testable hypotheses that can be verified using other investigatory techniques.

\section{Behavioral effects of cat odor and midazolam}

In presenting the cat odor stimulus in a confined space in which escape was not easily achieved, the present study differed from our previous mapping study (Dielenberg et al., 2001b). Under these confined conditions, a reluctance to contact the cat odor stimulus was evident, in line with previous reports involving varied testing environments (Blanchard et al., 1990; Zangrossi and File, 1992; McGregor et al., 2002; Li et al., 2004). In addition, cat odor disrupted ongoing "housekeeping" functions in rats, as evidenced by the inhibition of grooming behavior. More notably, cat odor promoted rearing, an effect we noted previously (Dielenberg et al., 2001a) and that is associated with vigilant scanning of the environment and increased distance from the cat odor stimulus.

As noted previously (Dielenberg et al., 1999; McGregor and Dielenberg, 1999; McGregor et al., 2002) midazolam altered the expression of defensive behavior in rats exposed to cat odor. Interpretation of this effect is complex because of the limited behavioral repertoire possible within the confined environment used here. Nonetheless, midazolam increased contact with the odor stimulus and prevented the high levels of rearing behavior that was evident in vehicle-treated rats. The effect on rearing might reflect a mild ataxic effect of the drug, although the dose used $(0.75 \mathrm{mg} / \mathrm{kg})$ is on the borderline of that affecting rotarod performance in rats (Drugan et al., 1996; Austin et al., 1999).

\section{Cat odor as a pheromone-like stimulus or kairomone}

Cat odor-exposed rats showed substantial activation in the posterior $\mathrm{AOB}$, suggesting processing by the accessory olfactory or vomeronasal system of the brain. A large body of work now shows that the behavioral response released by cat odor in rats is innate, powerful, and stereotypical in nature and seemingly occurs to minute quantities of an olfactory cue that are not detectable to the human nose. These characteristics are typical of other pheromones (Dulac and Torello, 2003) and support the hypothesis that cat odor may be processed as a pheromone-like stimulus.

The suggestion of vomeronasal involvement in the response to cat odor is supported by Panksepp (1998), who briefly reports that cat odor fails to disrupt play behavior in juvenile rats after sectioning of the vomeronasal nerve. In contrast, cat odor remained an effective anxiogenic stimulus when the main olfactory system was disrupted by the intranasal application of zinc sulfate (Panksepp, 1998).

Although the AOB has been traditionally implicated in the processing of within-species chemosensory cues, recent electrophysiological evidence has suggested sensitivity to a wider range of putative semiochemicals (Sam et al., 2001). The present results support this notion, indicating a major role for the $\mathrm{AOB}$ in pro- cessing chemical signals operating across species to elicit powerful instinctive responses.

Biologists use the term "kairomone" to refer to a pheromone or semiochemical that is released by one species that has a favorable adaptive effect on a different "receiving" species but no favorable effect on the transmitting species (Dicke and Grostal, 2001). It is interesting to ponder whether cat odor might qualify as a kairomone with reference to its effects on rats.

\section{The role of the main olfactory bulb}

Cat odor exposure was also associated with increased activation of the rat MOB, but this occurred against a backdrop of high basal MOB activation, and the cat odor effect was modest and not localized to a specific glomerular region. It is worth recalling that the cat odor stimulus was a collar that had been worn by a cat for at least 2 weeks, during which time the cat had presumably come into contact with a range of odorants within the house and garden in which it resides. There may also be many odorants present in the skin of the cat that do not have the same signaling function as the molecule (or molecules) that trigger the defensive response in rats. Thus, rats exposed to cat odor in the present study will no doubt have been exposed to a greater range of olfactory stimuli than those exposed to an unworn collar. Given this observation, and the results of Panksepp (1998) mentioned above, it is tempting to conclude that the cat odor stimulus is conveyed exclusively by mechanisms involving the AOB. However, additional work is clearly required to confirm this, particularly studies in which cat odor is presented after several hours of preexposure to clean air. This procedure would greatly lower basal MOB Fos expression and allow a clearer elucidation of any cat odor-induced activation $\mathrm{MOB}$ and in related sites, such as the AON.

\section{Region-specific effects of midazolam}

Midazolam had widespread inhibitory effects on cat odorinduced Fos expression, but, as Table 1 clearly shows, these effects were region specific. This agrees with a previous study, in which the benzodiazepine diazepam had region-specific effects on conditioned fear-induced c-fos mRNA in rats (Beck and Fibiger, 1995).

Given that midazolam was able to inhibit Fos expression in both the $\mathrm{AOB}$ and $\mathrm{MOB}$, it becomes critical to assess the extent to which the modulation of defensive behavior to cat odor by this drug reflects a simple drug-induced deficit in sensory processing. We believe that a sensory deficit is unlikely to explain the present results for several reasons: (1) the effect of midazolam on the $\mathrm{AOB}$ was limited to only the granule cell layer and did not involve the key output neurons of the mitral layer; (2) the major projection area of the AOB, the medial amygdala, showed only a marginal decrease in Fos expression with midazolam; and (3) several key structures involved in defensive behavior were activated during cat odor exposure in a midazolam-insensitive manner (e.g., the $\mathrm{VMH}$, paraventricular nucleus of the hypothalamus, PAG, and cuneiform nucleus). If midazolam had simply prevented olfactory processing of the cat odor stimulus, then a blanket reduction in Fos activation in defense-related sites would be expected.

A key action of benzodiazepines is to disinhibit approach in situations of fear or uncertainty and to promote risk assessment behavior at the expense of flight (Gray and McNaughton, 2000). The present results suggest that this shift in defensive behavior may involve an action on brain regions located at several levels of the defensive behavior hierarchy.

First, the medial prefrontal cortex may play a key role in coping with stress when it is inescapable, as in the present study, and, 
clearly, benzodiazepines have a potent capacity to deactivate such MPC stress-related circuitry. Second, the lateral septum (ventral part) may be critical in shifting the balance of defense toward passive rather than active responses (Mongeau et al., 2003) so that the strong inhibitory effect of benzodiazepines on this region may prevent immobility and promote more active response strategies.

Third, a modest action of benzodiazepines in the medial amygdala and BNST could reflect either a modulation of pheromonal signal processing or a specific modulation of unconditioned fear responses. Fourth, a specific action of benzodiazepines in the medial hypothalamic defense circuit, centered around the PMd, anterior hypothalamic, and preoptic areas, is evident, which attenuates the defensive response to specific predator cues. Presumably, for rodents to survive, they must have a mechanism that allows them to overcome innate fear response to predator cues so that foraging and other activities essential for survival can be resumed. An endogenous GABAergic ligand that allows habituation to predator cues may be active around the PMd and the medial hypothalamic circuit.

Finally, it is worth commenting further on the apparent insensitivity of the PAG to benzodiazepine actions. It is striking that a recent study on ultrasound-induced defense in mice noted that patterns of c-fos expression in the PAG did not readily differentiate between those mice that showed freezing from those that showed fleeing, whereas activation patterns in hypothalamic and limbic regions readily differentiated between these two response profiles (Mongeau et al., 2003). One can find parallels with the present results: clearly, benzodiazepines alter the behavioral strategy adopted toward a threat stimulus, but this appears to involve alterations at hypothalamic and forebrain levels rather than any readily observable modulation of brainstem function.

In agreement with this, it has been reported recently that dorsal PAG lesions only weakly affected cat odor-induced defensive behavior but had some modulation of its cardiovascular effects (Dielenberg et al., 2004). One other study indicated that benzodiazepines injected into the ventrolateral PAG failed to affect conditioned fear but had a strong effect on conditioned hypoalgesia (Harris and Westbrook, 1995). This raises the possibility that the engagement of PAG substrates by predatory cues may be preferentially related to possible autonomic and analgesic properties of these cues rather than the organization and triggering of specific defensive behaviors.

\section{References}

Allen KV, McGregor IS, Hunt GE, Singh ME, Mallet PE (2003) Regional differences in naloxone modulation of Delta(9)-THC induced Fos expression in rat brain. Neuropharmacology 44:264-274.

Austin M, Myles V, Brown PL, Mammola B, Drugan RC (1999) FG 7142and restraint-induced alterations in the ataxic effects of alcohol and midazolam in rats are time dependent. Pharmacol Biochem Behav 62:45-51.

Bachtell RK, Weitemier AZ, Galvan-Rosas A, Tsivkovskaia NO, Risinger FO, Phillips TJ, Grahame NJ, Ryabinin AE (2003) The Edinger-Westphallateral septum urocortin pathway and its relationship to alcohol consumption. J Neurosci 23:2477-2487.

Beck CH, Fibiger HC (1995) Conditioned fear-induced changes in behavior and in the expression of the immediate early gene c-fos: with and without diazepam pretreatment. J Neurosci 15:709-720.

Berridge CW, Mitton E, Clark W, Roth RH (1999) Engagement in a nonescape (displacement) behavior elicits a selective and lateralized suppression of frontal cortical dopaminergic utilization in stress. Synapse 32:187-197.

Blanchard DC, Blanchard RJ (1999) Cocaine potentiates defensive behaviors related to fear and anxiety. Neurosci Biobehav Rev 23:981-991.

Blanchard DC, Hynd AL, Minke KA, Minemoto T, Blanchard RJ (2001) Human defensive behaviors to threat scenarios show parallels to fear- and anxiety-related defense patterns of non-human mammals. Neurosci Biobehav Rev 25:761-770.

Blanchard DC, Li CI, Hubbard D, Markham CM, Yang M, Takahashi LK, Blanchard RJ (2003) Dorsal premammillary nucleus differentially modulates defensive behaviors induced by different threat stimuli in rats. Neurosci Lett 345:145-148.

Blanchard RJ, Blanchard DC, Weiss SM, Meyer S (1990) The effects of ethanol and diazepam on reactions to predatory odors. Pharmacol Biochem Behav 35:775-780.

Canteras NS (2002) The medial hypothalamic defensive system: hodological organization and functional implications. Pharmacol Biochem Behav 71:481-491.

Canteras NS, Goto M (1999) Fos-like immunoreactivity in the periaqueductal gray of rats exposed to a natural predator. NeuroReport 10:413-418.

Canteras NS, Chiavegatto S, Ribeiro Do Valle LE, Swanson LW (1997) Severe reduction of rat defensive behavior to a predator by discrete hypothalamic chemical lesions. Brain Res Bull 44:297-305.

Dayas CV, Buller KM, Day TA (1999) Neuroendocrine responses to an emotional stressor: evidence for involvement of the medial but not the central amygdala. Eur J Neurosci 11:2312-2322.

Dicke M, Grostal P (2001) Chemical detection of natural enemies by arthropods: an ecological perspective. Annu Rev Ecol Syst 32:1-23.

Dielenberg RA, Arnold JC, McGregor IS (1999) Low-dose midazolam attenuates predatory odor avoidance in rats. Pharmacol Biochem Behav 62:197-201.

Dielenberg RA, Carrive P, McGregor IS (2001a) The cardiovascular and behavioral response to cat odor in rats: unconditioned and conditioned effects. Brain Res 897:228-237.

Dielenberg RA, Hunt GE, McGregor IS (2001b) "When a rat smells a cat": the distribution of c-fos expression in the rat brain following exposure to a predatory odor. Neuroscience 104:1085-1097.

Dielenberg RA, Leman S, Carrive P (2004) Effect of dorsal periaqueductal gray lesions on cardiovascular and behavioral responses to cat odor exposure in rats. Behav Brain Res, in press.

Drugan RC, Coyle TS, Healy DJ, Chen S (1996) Stress controllability influences the ataxic properties of both ethanol and midazolam in the rat. Behav Neurosci 110:360-367.

Dulac C, Torello AT (2003) Molecular detection of pheromone signals in mammals: from genes to behaviour. Nat Rev Neurosci 4:551-562.

Espejo EF (1997) Selective dopamine depletion within the medial prefrontal cortex induces anxiogenic-like effects in rats placed on the elevated plus maze. Brain Res 762:281-284.

Fendt M, Endres T, Apfelbach R (2003) Temporary inactivation of the bed nucleus of the stria terminalis but not of the amygdala blocks freezing induced by trimethylthiazoline, a component of fox feces. J Neurosci 23:23-28.

Figueiredo HF, Bodie BL, Tauchi M, Dolgas CM, Herman JP (2003) Stress integration after acute and chronic predator stress: differential activation of central stress circuitry and sensitization of the hypothalamo-pituitaryadrenocortical axis. Endocrinology 144:5249-5258.

Fritschy JM, Mohler H (1995) GABAA-receptor heterogeneity in the adult rat brain: differential regional and cellular distribution of seven major subunits. J Comp Neurol 359:154-194.

Funk D, Amir S (2000) Circadian modulation of Fos responses to odor of the red fox, a rodent predator, in the rat olfactory system. Brain Res 866:262-267.

Gray JA, McNaughton N (2000) The neuropsychology of anxiety. Oxford: Oxford UP.

Guthrie KM, Gall C (2003) Anatomic mapping of neuronal odor responses in the developing rat olfactory bulb. J Comp Neurol 455:56-71.

Halpern M, Martinez-Marcos A (2003) Structure and function of the vomeronasal system: an update. Prog Neurobiol 70:245-318.

Harris JA, Westbrook RF (1995) Effects of benzodiazepine microinjection into the amygdala or periaqueductal gray on the expression of conditioned fear and hypoalgesia in rats. Behav Neurosci 109:295-304.

Higgs S, Cooper SJ (1996) Hyperphagia induced by direct administration of midazolam into the parabrachial nucleus of the rat. Eur J Pharmacol 313:1-9.

Johnson BA, Ho SL, Xu Z, Yihan JS, Yip S, Hingco EE, Leon M (2002) Functional mapping of the rat olfactory bulb using diverse odorants reveals modular responses to functional groups and hydrocarbon structural features. J Comp Neurol 449:180-194. 
Kaufmann WA, Humpel C, Alheid GF, Marksteiner J (2003) Compartmentation of alpha 1 and alpha $2 \mathrm{GABA}(\mathrm{A})$ receptor subunits within rat extended amygdala: implications for benzodiazepine action. Brain Res 964:91-99.

Keay KA, Bandler R (2001) Parallel circuits mediating distinct emotional coping reactions to different types of stress. Neurosci Biobehav Rev 25:669-678.

Klintsova AY, Philpot BD, Brunjes PC (1995) Fos protein immunoreactivity in the developing olfactory bulbs of normal and naris-occluded rats. Brain Res Dev Brain Res 86:114-122.

Kumar A, Dudley CA, Moss RL (1999) Functional dichotomy within the vomeronasal system: distinct zones of neuronal activity in the accessory olfactory bulb correlate with sex-specific behaviors. J Neurosci 19:RC32(1-6).

Laurie DJ, Seeburg PH, Wisden W (1992) The distribution of $13 \mathrm{GABA}_{\mathrm{A}}$ receptor subunit mRNAs in the rat brain. II. Olfactory bulb and cerebellum. J Neurosci 12:1063-1076.

Lee Y, Davis M (1997) Role of the hippocampus, the bed nucleus of the stria terminalis, and the amygdala in the excitatory effect of corticotropinreleasing hormone on the acoustic startle reflex. J Neurosci 17:6434-6446.

Li AB, Maglinao CD, Takahashi LK (2004) Medial amygdala modulation of predator odor-induced unconditioned fear in the rat. Behav Neurosci, in press.

McGregor IS, Dielenberg RA (1999) Differential anxiolytic efficacy of a benzodiazepine on first versus second exposure to a predatory odor in rats. Psychopharmacology 147:174-181.

McGregor IS, Schrama L, Ambermoon P, Dielenberg RA (2002) Not all "predator odors" are equal: cat odor but not 2,4,5 trimethylthiazoline (TMT; fox odor) elicits specific defensive behaviours in rats. Behav Brain Res 129:1-16

Menard J, Treit D (1999) Effects of centrally administered anxiolytic compounds in animal models of anxiety. Neurosci Biobehav Rev 23:591-613.

Milani H, Graeff FG (1987) GABA-benzodiazepine modulation of aversion in the medial hypothalamus of the rat. Pharmacol Biochem Behav 28:21-27.
Mongeau R, Miller GA, Chiang E, Anderson DJ (2003) Neural correlates of competing fear behaviors evoked by an innately aversive stimulus. J Neurosci 23:3855-3868.

Panksepp J (1998) Affective neuroscience, pp 221-222. New York: Oxford UP.

Paxinos G, Watson C (1997) The rat brain in stereotaxic coordinates, Ed 3. Sydney: Academic.

Quaglino E, Giustetto M, Panzanelli P, Cantino D, Fasolo A, Sassoe-Pognetto M (1999) Immunocytochemical localization of glutamate and gammaaminobutyric acid in the accessory olfactory bulb of the rat. J Comp Neurol 408:61-72.

Russo AS, Guimaraes FS, De Aguiar JC, Graeff FG (1993) Role of benzodiazepine receptors located in the dorsal periaqueductal grey of rats in anxiety. Psychopharmacology (Berl) 110:198-202.

Sallaz M, Jourdan F (1993) C-fos expression and 2-deoxyglucose uptake in the olfactory bulb of odour-stimulated awake rats. NeuroReport 4:55-58.

Sam M, Vora S, Malnic B, Ma W, Novotny MV, Buck LB (2001) Neuropharmacology. Odorants may arouse instinctive behaviours. Nature 412:142.

Scalia F, Winans SS (1975) The differential projections of the olfactory bulb and accessory olfactory bulb in mammals. J Comp Neurol 161:31-55.

Shipley MT, McLean JH, Ennis M (1995) The olfactory system. In: The rat nervous system, Ed 2 (Paxinos G, ed), pp 899-926. Sydney: Academic.

Singewald N, Salchner P, Sharp T (2003) Induction of c-Fos expression in specific areas of the fear circuitry in rat forebrain by anxiogenic drugs. Biol Psychiatry 53:275-283.

Stephenson CP, Hunt GE, Topple AN, McGregor IS (1999) The distribution of 3,4-methylenedioxymethamphetamine "Ecstasy"-induced c-fos expression in rat brain. Neuroscience 92:1011-1023.

Walker DL, Toufexis DJ, Davis M (2003) Role of the bed nucleus of the stria terminalis versus the amygdala in fear, stress, and anxiety. Eur J Pharmacol 463:199-216.

Zangrossi Jr H, File SE (1992) Chlordiazepoxide reduces the generalised anxiety, but not the direct responses, of rats exposed to cat odor. Pharmacol Biochem Behav 43:1195-1200. 